\title{
CON: Propofol Is Better Than Etomidate for Induction in Cardiac Surgical Patients
}

\author{
Suruchi Ladha ${ }^{1}$ Arvind Prakash ${ }^{1}$ \\ ${ }^{1}$ Department of Cardiac Anaesthesia, National Heart \\ Institute 49-50, Community Centre, New Delhi, India
}

\author{
Address for correspondence Suruchi Ladha, DM, Department of \\ Cardiac Anesthesia, National Heart Institute 49-50, Community \\ Centre, East of Kailash, New Delhi - 1100 65, India \\ (e-mail: suruchi.ladha@gmail.com).
}
Abstract
Keywords
- cardiac surgical
- etomidate
- induction
- propofol

There is an ongoing debate regarding the choice of ideal induction agent between propofol and etomidate for cardiac surgical patients. Etomidate appears to be better than propofol as the induction agent due to the superior hemodynamic stability. However, the adrenal suppression due to etomidate can pose challenges to the anesthesiologist in certain clinical situations.

\section{Introduction}

The choice of an ideal induction agent in cardiac surgical patients is not always straight forward. The comparison between propofol and etomidate as the preferred induction agent in cardiac surgical patients is difficult and the evidence is varied. There are only a few published studies comparing the perioperative effects of etomidate and propofol in cardiac surgical patients. ${ }^{1,2}$ The evidence in this area is conflicting, possibly reflecting different experimental setups along with variations in anesthetic techniques, drug dosages, and variations in timing and techniques of measuring clinical data. ${ }^{3,4}$ Furthermore, a few studies have some degree of methodological drawbacks such as retrospective design, selection biases, small sample sizes, and failure to blinding.

Propofol is undoubtedly one of the most popular intravenous anesthetic agents for induction and maintenance of anesthesia. ${ }^{5,6}$ In a recent survey, propofol in combination with an opioid was the most popular choice of anesthetic techniques for off-pump coronary artery bypass grafting operation. ${ }^{7}$ However, etomidate is an induction agent known for its smooth intubating conditions and cardiovascular stability. It has a very high therapeutic index of safety, among all the other available induction agents. It has a favorable kinetic profile of producing rapid onset and offset of action. In comparative studies with other anesthetic drugs, etomidate is usually described as the drug that causes the least changes in all hemodynamic variables. ${ }^{12,8}$ Patients who have hypovolemia, cardiac tamponade, or low cardiac output probably represent the population for whom etomidate is better than propofol. In certain situations, the advantages of etomidate outweigh those of propofol like emergency use situations in which rapid induction is essential in cardiac surgical patients. ${ }^{9}$

Various studies have evaluated the hemodynamic effect of propofol and etomidate. ${ }^{1-3}$ The hemodynamic effects of propofol have been investigated in American Society of Anesthesiologists class I (normal and healthy) and class II (mild systemic disease) patients, elderly patients, patients with coronary artery disease and good left ventricular function, and patients with impaired left ventricular function. Most studies have demonstrated significant reductions in systemic vascular resistance (SVR) (9-30\%), cardiac index (CI), stroke volume, and left ventricular stroke work index after propofol. Although controversial, the evidence points to a dose-dependent decrease in myocardial contractility. ${ }^{2}$ Transient hypotension after propofol administration is common. Most anesthesiologist can manage this hypotension published online March 27, 2021
DOI https://doi.org/

$10.1055 / \mathrm{s}-0041-1728957$ ISSN 2457-0206.
(C) 2021. Official Publication of The Simulation Society (TSS), accredited by International Society of Cardiovascular Ultrasound (ISCU).

This is an open access article published by Thieme under the terms of the Creative Commons Attribution-NonDerivative-NonCommercial-License, permitting copying and reproduction so long as the original work is given appropriate credit. Contents may not be used for commercial purposes, or adapted, remixed, transformed or built upon. (https://creativecommons.org/licenses/by-nc-nd/4.0/) Thieme Medical and Scientific Publishers Pvt. Ltd., A-12, 2nd Floor, Sector 2, Noida-201301 UP, India 
with fluids and small dose of vasopressors. However, the effects of such postinduction hypotension on patient outcome need to be determined. Reich et al observed in a study including 2,406 patients that those who had postinduction hypotension had prolonged postoperative stay and/or death in comparison to those without postinduction hypotension. It was concluded in this study that propofol be avoided for induction in patients who present with baseline mean arterial pressure (MAP) $<70$ $\mathrm{mm} \mathrm{Hg}$ and to avoid severe hypotension, alternatives to propofol anesthetic induction be considered like etomidate. ${ }^{10}$ Alcock et al found that in patients treated with antiplatelet agents for cardiovascular comorbidities undergoing major noncardiac surgery, intraoperative hypotension was identified as one of three independent predictors of intraoperative myocardial ischemia and necrosis assessed using postoperative troponin $\mathrm{T}$ levels. ${ }^{11}$ Given the high risk of poor outcome after postinduction and intraoperative hypotension, the ideal induction agent would avoid any major deviation from baseline hemodynamic parameters. ${ }^{1}$ The use of etomidate in doses of 0.15 to $0.30 \mathrm{mg} /$ $\mathrm{kg}$ both in healthy subjects or patients who have compensated ischemic heart disease did not produce any significant change in variables such as heart rate, pulmonary artery pressure, pulmonary capillary wedge pressure, left ventricular end-diastolic pressure, right artery pressure, CI, SVR, pulmonary vascular resistance, and left ventricular $\mathrm{dP} / \mathrm{dt}$. Compared with other anesthetic agents, etomidate produces the least change in the balance of myocardial oxygen demand and supply. ${ }^{12}$ Systemic blood pressure remains unchanged in most studies. In a randomized controlled trial by Hannam et al found that propofol caused a 34\% greater reduction in MAP-time integral from baseline after induction of anesthesia than etomidate, despite more frequent use of vasopressors with propofol, confirming the superior hemodynamic profile of etomidate in this context. ${ }^{1}$ Haessler et al compared the effect of etomidate with propofol combined with fentanyl, respectively, in patients with severe coronary artery disease. The study was terminated early owing to myocardial ischemia in two patients who were induced with propofol. ${ }^{13}$ Seitz et al randomly assigned 20 patients scheduled for coronary artery bypass grafting to induction with propofol and fentanyl or etomidate and fentanyl plus midazolam. Even with a slow titration of propofol, 5 of 10 patients needed active treatment for hypotensive episodes. ${ }^{14}$

In patients with valvular heart lesions, the maintenance of preload is desired for stenotic and regurgitant lesions. Preservation or even increasing afterload is the therapeutic goal for stenotic lesions, such as aortic stenosis, hypertrophic obstructive cardiomyopathy, and mitral stenosis. Etomidate in comparison to propofol would appear to be the ideal induction agent in such patients. In a randomized controlled, double-blind, prospective study of anesthesia induction, in patients with severe aortic stenosis by Bendell et al, it was found that propofol is twice as likely as etomidate to evoke hypotension in anesthesia induction with severe aortic stenosis. ${ }^{3}$ Propofol can have negative effect on the myocardial contractility but etomidate does not have such negative effect.

Patients with congestive heart failure have two specific concerns: they are dependent on high sympathetic tone and often are treated with angiotensin-converting enzyme inhibitors and angiotensin-receptor blockers. ${ }^{15}$ Angiotensin-converting enzyme inhibitors decrease the sympathetic nervous system reactivity and put patients at high risk of postinduction hypotension. Compared with propofol, etomidate has been shown to maintain the sympathetic tone after usual induction doses, whereas propofol decreases this tone. Ebert et al studied the sympathetic response to induction of anesthesia with propofol and etomidate by using microneurography to measure the sympathetic tone..$^{16}$ Propofol-induced hypotension is mediated by inhibition of sympathetic nervous system and impairment of baroreflex regulatory mechanism. Etomidate maintains hemodynamic stability through preservation of both sympathetic outflow and autonomic reflexes. There is, however, emerging evidence that propofol may enhance antioxidant activity in the heart and may prevent lipid peroxidation after ischemia and reperfusion, offering a potential protection of the heart. There is no evidence of such role by etomidate.

Hence, it is an established fact that etomidate often is the favored induction agent for patients who are hemodynamically compromised because of its relative cardiovascular stability. However, there is ongoing debate about its use in critically ill patients because of its inhibition of adrenal steroidogenesis. ${ }^{6 .}{ }^{9}$ Etomidate infusion and single injections directly suppress adrenocortical function, which interferes with the normal stress response. ${ }^{17}$ Blockade of $11 \beta$-hydroxylation mediated by the imidazole radical of etomidate results in decreased biosynthesis of cortisol and aldosterone. ${ }^{18,19}$ There is universal agreement and strong evidence that etomidate causes adrenal suppression even at low blood levels and even after a single bolus. There is also reasonable evidence that the duration of adrenal suppression lasts significantly longer than the hypnotic effect. However, the intensity of this suppression and its clinical significance remain inconsistent and inadequately quantified in cardiac surgery. Several studies have observed that due to the adrenal steroid synthesis inhibition by etomidate, its use in trauma patients or patients with sepsis translates to increase in morbidity and mortality. ${ }^{17-20}$ Such patients often have a minimal physiologic reserve and may develop hemodynamic perturbations because of suppression of the adrenal axis. Cardiac surgery and the initiation of cardiopulmonary bypass are known stimulators of the inflammatory response. ${ }^{21}$ Catecholamine and stress hormone levels are increased in patients undergoing bypass. These endogenous cytokines, such as cortisol, are thought to play a role in the maintenance of vascular tone. The anesthetist using etomidate often wonders whether the impairment of these stress hormones will contribute to the increase in the vasopressor/inotropic requirement in the postoperative period, will the consequences of vasoconstriction, such as ischemia, lead to increased morbidity, will the duration of mechanical ventilation increase, will there be more ischemia-reperfusion injury, and ultimately will all this lead to increased mortality.

Few studies have found that the use of etomidate was associated with a substantially increased risk for 30-day mortality, cardiovascular morbidity, and prolonged hospital stay in noncardiac surgery like Komatsu et al, but the factors in cardiac surgery vary from that in noncardiac surgery.2 ${ }^{22}$ The evidence of postoperative adverse outcomes by using etomidate as an induction agent in noncardiac surgery cannot be extrapolated 
to cardiac surgery. Wagneret al studied the postoperative outcomes of the use of etomidate in patients undergoing cardiac surgery and found that single use etomidate is not associated with severe hypotension, longer mechanical ventilation hours, longer length of hospital stay, or in-hospital mortality. ${ }^{23}$ Basciani et al found that in patients undergoing elective cardiac surgery, laboratory indicators of etomidate-induced adrenal insufficiency do not translate into increased vasopressor requirement or inferior early outcomes. ${ }^{8}$ Similarly, Komatsu et al found that etomidate was not associated with increased incidence of postoperative atrial arrhythmia or increased intensive care unit or hospital stay. ${ }^{24}$ Evidence for postoperative systemic inflammatory response syndrome (SIRS) due to adrenal insufficiency is of particular relevance for patients after on-pump cardiac surgery. It is well known that on-pump cardiac surgery per se is a risk factor for postoperative SIRS and infection. Heinrich et al found that there is no evidence for differences in key clinical outcome in cardiac surgery patients based on anesthesia induction with or without administration of a single dose of etomidate. ${ }^{4}$

\section{Summary}

Etomidate appears to be better than propofol as the induction agent due to the superior hemodynamic stability. Hypotension after induction of patients with valvular heart disease and congestive heart failure has been associated with increased morbidity and mortality. In the current era, when the cardiac anesthesiologist encounters patients with severe comorbidities, the maintenance of a stable hemodynamic profile during the induction of anesthesia is very important. Although it is established that etomidate causes adrenal suppression, there is little evidence for differences in key clinical outcome in cardiac surgical patients based on anesthesia induction with a single dose of etomidate.

\section{Conflict of Interest}

None.

\section{References}

1 Hannam JA, Mitchell SJ, Cumin D, et al. Haemodynamic profiles of etomidate vs propofol for induction of anaesthesia: a randomised controlled trial in patients undergoing cardiac surgery. Br J Anaesth 2019;122(2):198-205

2 Soleimani A, Heidari N, Habibi MR, et al. Comparing hemodynamic responses to diazepam, propofol and etomidate during anesthesia induction in patients with left ventricular dysfunction undergoing coronary artery bypass graft surgery: a double-blind, randomized clinical trial. Med Arh 2017;71(3):198-203

3 Bendel S, Ruokonen E, Pölönen P, Uusaro A. Propofol causes more hypotension than etomidate in patients with severe aortic stenosis: a double-blind, randomized study comparing propofol and etomidate. Acta Anaesthesiol Scand 2007;51(3):284-289

4 Heinrich S, Schmidt J, Ackermann A, Moritz A, Harig F, Castellanos I. Comparison of clinical outcome variables in patients with and without etomidate-facilitated anesthesia induction ahead of major cardiac surgery: a retrospective analysis. Crit Care 2014;18(4):R150 10.1186/cc13988

5 KrzychLJ,SzurlejD, BochenekA. Rationalefor propofol use incardiac surgery. J Cardiothorac Vasc Anesth 2009;23(6):878-885
6 Malhotra AK. Con: etomidate-the ideal induction agent for a cardiac anesthetic? JCardiothorac Vasc Anesth 2013;27(1):178-179

7 Chassot PG, van der Linden P, Zaugg M, Mueller XM, Spahn DR. Off-pump coronary artery bypass surgery: physiology and anaesthetic management. Br J Anaesth 2004;92(3):400-413

8 Basciani RM, Rindlisbacher A, Begert E, et al. Anaesthetic induction with etomidate in cardiac surgery: a randomised controlled trial. Eur J Anaesthesiol 2016;33(6):417-424

9 Budde AO, Mets B. Pro: etomidate is the ideal induction agent for a cardiacanesthetic.JCardiothoracVasc Anesth2013;27(1):180-183

10 Reich DL, Hossain S, Krol M, et al. Predictors of hypotension after induction of general anesthesia. Anesth Analg 2005;101(3):622-628

11 Alcock RF, Kouzios D, Naoum C, Hillis GS, Brieger DB. Perioperative myocardial necrosis in patients at high cardiovascular risk undergoing elective non-cardiac surgery. Heart 2012;98(10):792-798

12 Morel J, Salard M, Castelain C, et al. Haemodynamic consequences of etomidate administration in elective cardiac surgery: a randomized double-blinded study. Br J Anaesth 2011;107(4):503-509

13 Haessler R, Madler C, Klasing S, Schwender D, Peter K. Propofol/ fentanyl versus etomidate/fentanyl for the induction of anesthesia in patients with aortic insufficiency and coronary artery disease. J Cardiothorac Vasc Anesth 1992;6(2):173-180

14 Seitz W, Lübbe N, Schaps D, Haverich A, Kirchner E. [Propofol for induction and maintenance of anesthesia during heart surgery. Results of pharmacological studies in man]. Anaesthesist 1991;40(3):145-152

15 Groban L, Butterworth J. Perioperative management of chronic heart failure. Anesth Analg 2006;103(3):557-575

16 Ebert TJ, Muzi M, Berens R, Goff D, Kampine JP. Sympathetic responses to induction of anesthesia in humans with propofol or etomidate. Anesthesiology 1992;76(5):725-733

17 Cherfan AJ, Arabi YM, Al-Dorzi HM, Kenny LP. Advantages and disadvantages of etomidate use for intubation of patients with sepsis. Pharmacotherapy 2012;32(5):475-482

18 Bruder EA, Ball IM, Ridi S, Pickett W, Hohl C. Single induction dose of etomidate versus other induction agents for endotracheal intubation in critically ill patients. Cochrane Database Syst Rev 2015;1(1):CD010225 10.1002/14651858. CD010225.pub2

19 Kulstad EB, Kalimullah EA, Tekwani KL, Courtney DM. Etomidate as an induction agent in septic patients: red flags or false alarms? West J Emerg Med 2010;11(2):161-172

20 Hinkewich C, Green R. The impact of etomidate on mortality in trauma patients. Can J Anaesth 2014;61(7):650-655

21 Iribarren JL, Jiménez JJ, Hernández D, et al. Relative adrenal insufficiency and hemodynamic status in cardiopulmonary bypass surgery patients. A prospective cohort study. J Cardiothorac Surg 2010;5:26-32 Budde AO, Mets B

22 Komatsu R, You J, Mascha EJ, Sessler DI, Kasuya Y, Turan A. Anesthetic induction with etomidate, rather than propofol, is associated with increased 30-day mortality and cardiovascular morbidity after noncardiac surgery. Anesth Analg 2013;117(6):1329-1337

23 Wagner CE, Bick JS, Johnson D, et al. Etomidate use and postoperative outcomes among cardiac surgery patients. Anesthesiology 2014;120(3):579-589

24 Komatsu R, Makarova N, You J, et al. Etomidate and the risk of complications after cardiac surgery: a retrospective cohort analysis. J Cardiothorac Vasc Anesth 2016;30(6):1516-1522 